\title{
Hidrología y crecimiento de una plantación de cerezo para madera de calidad en el mediterráneo: papel del tipo de suelo, el riego y el laboreo
}

\author{
Molina, A.J. ${ }^{*}$, Llorens, P. $^{2}$, Aranda, X' ${ }^{1}$, Savé, R. ${ }^{1}$, Biel, C. ${ }^{1}$ \\ 'IRTA, Torre Marimón, Caldes de Montbuí, Barcelona \\ ${ }^{2} 2$ Institut de Diagnosi Ambiental i Estudis de l'Aigua (IDAEA)-CSIC, Barcelona \\ "e-mail: antonio.molina@irta.cat
}

\section{Resumen}

Las plantaciones de frondosas para madera de calidad están creciendo progresivamente en nuestro país como consecuencia de su alto valor de mercado y de una demanda no satisfecha con la producción propia. Sin embargo, la mayor parte de éstas se encuentran en mal estado como consecuencia de varios factores, siendo los más importantes una mala elección del material genético, del sitio de plantación y/o una inadecuada gestión selvícola. Dentro de este contexto, el objetivo de este trabajo es evaluar el efecto del tipo de suelo, el laboreo y del riego en el crecimiento, los flujos hídricos y la respuesta fisiológica de los árboles en una plantación de cerezo. La plantación estudiada está ubicada en Caldes de Montbuí (41 $\left.36^{\prime} 47^{\prime \prime} \mathrm{N}, 2^{\circ} 10^{\prime} 11^{\prime \prime} \mathrm{E}\right)$, con clima mediterráneo, con precipitación acumulada y temperatura medias anuales de $600 \mathrm{~mm}$ y $15^{\circ} \mathrm{C}$ respectivamente.

Los resultados indican que la cubierta ha tenido muy poco efecto en la partición de la lluvia, con un valor de interceptación de un 3\% de la precipitación. En lo que se refiere al papel del tipo de suelo, el laboreo y el riego, los resultados han sido diferentes dependiendo de la variable analizada y de la escala de tiempo considerada. Por ejemplo, el factor que más ha afectado a la transpiración ha sido el tipo de suelo, con una diferencia del 50\% entre los árboles regados. En cambio, el crecimiento acumulado en diámetro de la plantación se ha visto afectado por todos los factores analizados.

Palabras clave: Hidrología, madera noble, nogal, transpiración. 


\section{Introducción}

Las plantaciones de frondosas para madera de calidad se han incrementado en los últimos años en Europa (Montero et al., 2003) como consecuencia, por una parte, de una demanda permanente no satisfecha por la producción propia y, por otra, de los incentivos de la Unión Europa para promover su establecimiento gracias a su importante papel en la captación del carbono atmosférico (Cambria and Pierangeli, 2012). A su vez, y como aspecto quizá más relevante de cara a los productores, es que pueden ser cultivos complementarios de alta rentabilidad, consistentes normalmente en manchas de bosquetes dispersos dentro del mosaico agrícola.

En nuestro país, la producción propia de madera de calidad se encontraba bien representada antes de los años 50, sobre todo con ejemplares de carácter natural de cerezo y nogal. Sin embargo, tras la corta de éstos, la escasa reforestación llevada a cabo con estas especies trajo consigo un déficit importante en los años posteriores. Un punto y aparte supuso el programa de Reforestación de Tierras Agrarias, gracias al cual dichas plantaciones se han retomado en los últimos 15 años (Vilanova, comunicación personal) generalmente en regiones despobladas con suelos poco fértiles, donde las actividades agrarias normales no han sido viables (MAPA, 2006).

En la actualidad, España cuenta como mínimo con una superficie de 8000 ha para producción de madera calidad (Aletà y Vilanova, 2014). Sin embargo, a pesar de que dichas plantaciones vayan en aumento en nuestro país, aún existe un déficit importante de información científica al respecto, sobre todo en lo referente a las necesidades hídricas de dichas plantaciones (Ripoll et al., 2013), su papel en el ciclo hidrológico local y la necesidad o no del laboreo para la mejora de la permeabilidad del suelo (Moreno et al., 1997), con la correspondiente eliminación de la vegetación herbácea por su presumible competencia con la plantación objetivo. Por un lado, los coeficientes de cultivo propios para una correcta estimación del riego no han sido aún determinados para estas plantaciones, por lo que se suelen utilizar los procedentes de plantaciones frutales (Allen et al., 2006). Por otro lado, existe poca información sobre el efecto del establecimiento y desarrollo de estas plantaciones en los flujos hídricos. Mientras que los cambios del tipo de cubierta vegetal han sido ampliamente estudiados en cuencas de cabecera (p. ej. Bosch and Hewlett, 1982, Brown et al., 2005), aún existen pocos trabajos que se focalicen en el establecimiento de plantaciones forestales dentro del mosaico agronómico, y por tanto no existe un consenso científico claro del papel de éstas en ciclo hidrológico local (Farley et al., 2005; Gliessman, 1990).

Este trabajo presenta los resultados de un proyecto de tres años de duración llevado a cabo en una plantación joven de cerezos para madera de calidad en el ámbito mediterráneo. Se evaluaron tres efectos clave para el buen desarrollo de la plantación: el tipo del suelo, el riego y la influencia del laboreo. Para su correcta evaluación, se llevaron a cabo mediciones relacionadas con el crecimiento de los árboles, de las características edáficas y de las relaciones árbol-agua. 


\section{Material y métodos}

\section{1. Área de estudio y diseño experimental}

El ensayo se ha llevado a cabo en las instalaciones del IRTA de Torre Marimón

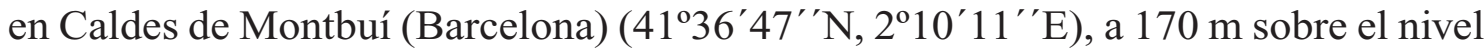
del mar. El clima de la zona es mediterráneo seco (Josa y Quintana, 2000), con precipitación acumulada y temperatura medias anuales de $600 \mathrm{~mm}$ y $15^{\circ} \mathrm{C}$ respectivamente, y evapotranspiración potencial acumulada de $847 \mathrm{~mm}$. El uso principal del suelo es forestal, con bosquetes dispersos de pino carrasco y piñonero, y en menor medida agrícola, con el forraje y las leguminosas ocupando un $50 \%$ de la superficie de la cuenca (Josa y Quintana, 2000).

La plantación de cerezo para madera de calidad estudiada tenía 8 años de edad al comienzo del experimento (2011) y se llevó a cabo con un clon procedente de Castilla La Mancha (CYL-01) a un marco de plantación de 4x4 metros (625 pies ha-1). El período de estudio fue de 3 años (2011-2013). Las características estructurales de la plantación al inicio del experimento están descritas en la Tab. 1 .

El diseño experimental fue de bloques completos al azar con 3 bloques o repeticiones. La disposición de éstos atendiendo a la presencia de un aluvión fluvial, con el bloque 1 el más influenciado por éste, el 2 con carácter de transición y el 3 el que menos. En el presente trabajo se presentan los resultados más contrastados, los de los bloques de peor y mejor calidad edáfica ( 1 y 3 respectivamente) (Tab. 1). Además del efecto bloque o tipo de suelo, se testó el efecto del riego y el laboreo.

Por tanto, cada bloque contaba con 4 unidades experimentales (12 en total) de 4 árboles estudiados cada una ( $64 \mathrm{~m}^{2}$ cada una), más los considerados como de efecto borde: "no laboreo+no riego" (NTNR), "no laboreo+riego" (NTR), "laboreo+no riego" (TNR) y "laboreo+riego" (TR).

Tabla 1. Variables descriptoras (media y desviación típica) del suelo y de la vegetación (DBH y altura medidos al inicio del experimento y cobertura durante el primer año) para 2 de los bloques estudiados. La cobertura se estimó gracias al empleo de fotos y su posterior procesado para determinar los porcentajes de los diferentes colores presentes

\begin{tabular}{ccccccc}
\hline Bloque & Textura & $\begin{array}{c}\% \\
\text { Elementos } \\
\text { gruesos }\end{array}$ & $\begin{array}{c}\text { Capacidad } \\
\text { Retención } \\
(\mathrm{mm})\end{array}$ & $\begin{array}{c}\text { DBH } \\
(\mathrm{cm})\end{array}$ & $\begin{array}{c}\text { Altura } \\
(\mathrm{m})\end{array}$ & $\begin{array}{c}\text { Cobertura } \\
(\%)\end{array}$ \\
\hline 1 & Franco-arenosa & $50 \pm 20$ & $37 \pm 8$ & $5 \pm 0,3$ & $4 \pm 0,2$ & $11 \pm 8$ \\
\hline 3 & Franco & $14 \pm 12$ & $102 \pm 5$ & $6 \pm 0,3$ & $5 \pm 0,3$ & $16 \pm 11$ \\
\hline
\end{tabular}

La dosis de riego de los tratamientos regados (R) se aplicaron durante los meses de mayo a septiembre, con 4 emisores por árbol $\left(161 \mathrm{~h}^{-1}\right.$ árbol $\left.{ }^{-1}\right)$ ubicados a 25 y 50 
$\mathrm{cm}$ del tronco en las líneas de riego orientadas de norte a sur. Las dosis diarias se calcularon al principio de cada semana, como el $60 \%$ de la evapotranspiración de la semana anterior y con coeficientes de cultivo propios de plantaciones frutales (Allen et $a l ., 2006)$. En este sentido, si la lluvia era mayor a la evapotranspiración ajustada, el contenido hídrico del suelo se consideraba suficiente y el riego no se aplicaba.

El laboreo del suelo (tratamientos TNR y TR) se llevó a cabo cada 3 a 4 meses con una fresadora hasta $30 \mathrm{~cm}$ de profundidad. Por el contrario, en los tratamientos no trabajados (NT) la vegetación espontánea se cortaba cada 2 meses en las líneas de riego y una vez al año (en el momento de máximo crecimiento) en toda la parcela, dejándola con aproximadamente $10 \mathrm{~cm}$ de altura.

Por último, se realizaron 2 podas durante el periodo de estudio, en junio de 2011 y 2013, encaminadas a la obtención de un fuste limpio de al menos $2 \mathrm{~m}$ de altura y siguiendo los procedimientos descritos para este tipo de plantaciones (Montero et al., 2003). Cada poda suponía la eliminación de aproximadamente un $25 \%$ del total de la biomasa del dosel.

\subsection{Mediciones relacionadas con el crecimiento y el carbono en el suelo}

Se llevó a cabo un seguimiento de las principales variables relacionadas con el crecimiento de los árboles durante el periodo de estudio, esto es, diámetro a la altura del pecho y altura total (mensual y anualmente, respectivamente), así como la cuantificación de la biomasa seca substraída del dosel por las podas y de la evolución de la cobertura forestal. Esta última se llevó a cabo mediante fotografías periódicas en 2 puntos situados a $50 \mathrm{~cm}$ al norte y sur de un árbol por cada unidad experimental, y siguiendo el procedimiento descrito en Casadesus et al. (2007) para el posterior procesado de las imágenes y el cálculo final de cobertura relativa (\%).

Por otra parte, la materia orgánica del suelo se aproximó mediante el carbono orgánico (método Walkley-Black). Se realizaron 2 calicatas en el centro de cada unidad experimental, una durante el verano de 2012 y otra al final del 2013. Las profundidades de muestreo fueron de 25, 50 y $100 \mathrm{~cm}$.

\subsection{Mediciones hidrológicas}

Las variables climáticas (humedad y temperatura del aire, velocidad y dirección del viento, precipitación y radiación solar y neta) se midieron en dos estaciones meteorológicas, una situada a $2 \mathrm{~m}$ sobre el dosel (a $8 \mathrm{~m}$ sobre el suelo) y otra en un claro muy cercano a la plantación (a $2 \mathrm{~m}$ sobre el suelo).

La trascolación, se midió con 58 pluviómetros registradores $(0.2 \mathrm{~mm}$ de resolución, Rain-o-Matic, Pronamic, Dinamarca) repartidos de forma aleatoria en un rectángulo de $128 \mathrm{~m}^{2}$ dentro de la plantación. Este diseño se llevó a cabo durante el segundo y tercer año de estudio ya que durante el primer año no se encontraron diferencias en la trascolación de las unidades experimentales (pluviómetros bajo copa, escala árbol) y por tanto se creyó más conveniente concentrar las mediciones en una subparcerla (escala parcela). La escorrentía cortical se midió en los 8 árboles com- 
prendidos en el rectángulo seleccionado, con 6 pluviómetros registradores $(0.2 \mathrm{~mm}$ de resolución, Rain-o-Matic, Pronamic, Dinamarca) y 2 depósitos totalizadores de 10 1 de capacidad.

La transpiración se midió con 12 sensores de flujo de savia (HRM, ICT International, Australia) basados en el método del pulso de calor (Burgess et al., 2001). En cada una de las 12 unidades experimentales, se seleccionó un árbol de los 4 monitoreados para el crecimiento y el sensor se dispuso a la altura del pecho en la orientación este. La transpiración por árbol se estimó posteriormente teniendo en cuenta la albura de cada árbol, estimada por el cambio de color entre albura y duramen en cores extraídos y con la inyección de colorante (ácido fucsínico a $0.1 \%$ ) siguiendo las indicaciones de Umebayashi et al. (2007). Para estimar la transpiración de la parcela se consideró la densidad de la plantación de 625 pies ha $^{-1}$.

Por último, en cada unidad experimental se dispusieron 5 sensores para medir el contenido hídrico del suelo (10-HS, Decagon Devices, USA), 3 bajo influencia de copa (a 25, 50 y $100 \mathrm{~cm}$ de profundidad) y 2 en el centro de ésta (a 25 y $50 \mathrm{~cm}$ de profundidad), resultando en un total de 60 sensores $(12 \times 5)$.

\subsection{Análisis estadístico}

Los 3 factores testados se evaluaron con el procedimiento de modelo lineal general univariante, con los 3 factores considerados como fijos y considerando la interacción de los factores "tipo de suelo" con "riego" y con "laboreo", respectivamente. Previamente a la comparación de medias, se estudió la normalidad de los datos y la homogeneidad de las varianzas mediante los test de Kolmogorv-Smirnoff y Levene, respectivamente. Los análisis se llevaron a cabo mediante el paquete estadístico SPSS 16.0 .

\section{Resultados}

\subsection{Crecimiento y carbono en el suelo}

Durante los 3 años de estudio, los árboles experimentaron incrementos de diámetro y altura muy dispares, con rangos que fueron desde 1,5 a 5,7 cm y desde 0,06 a $2,13 \mathrm{~m}$ respectivamente (Fig. 1). Esta variabilidad vino dada por la influencia de los tres factores testados que tuvieron efectos significativos $(p<0.001)$ en ambas variables según el análisis de la varianza correspondiente. El efecto del tipo de suelo explicó la mayor parte de la variabilidad y el laboreo la menor (por ejemplo, $F=79,2$ frente a $\mathrm{F}=9,2$ para la variable incremento de diámetro). Dicho análisis también mostró no interacción entre los factores, indicando a su vez que tanto el riego como el laboreo no supusieron efectos diferentes según el tipo de suelo.

La figura 2 presenta la evolución de la cobertura y el efecto de las dos podas en ésta para las unidades experimentales más dispares del experimento, esto es, el tra- 


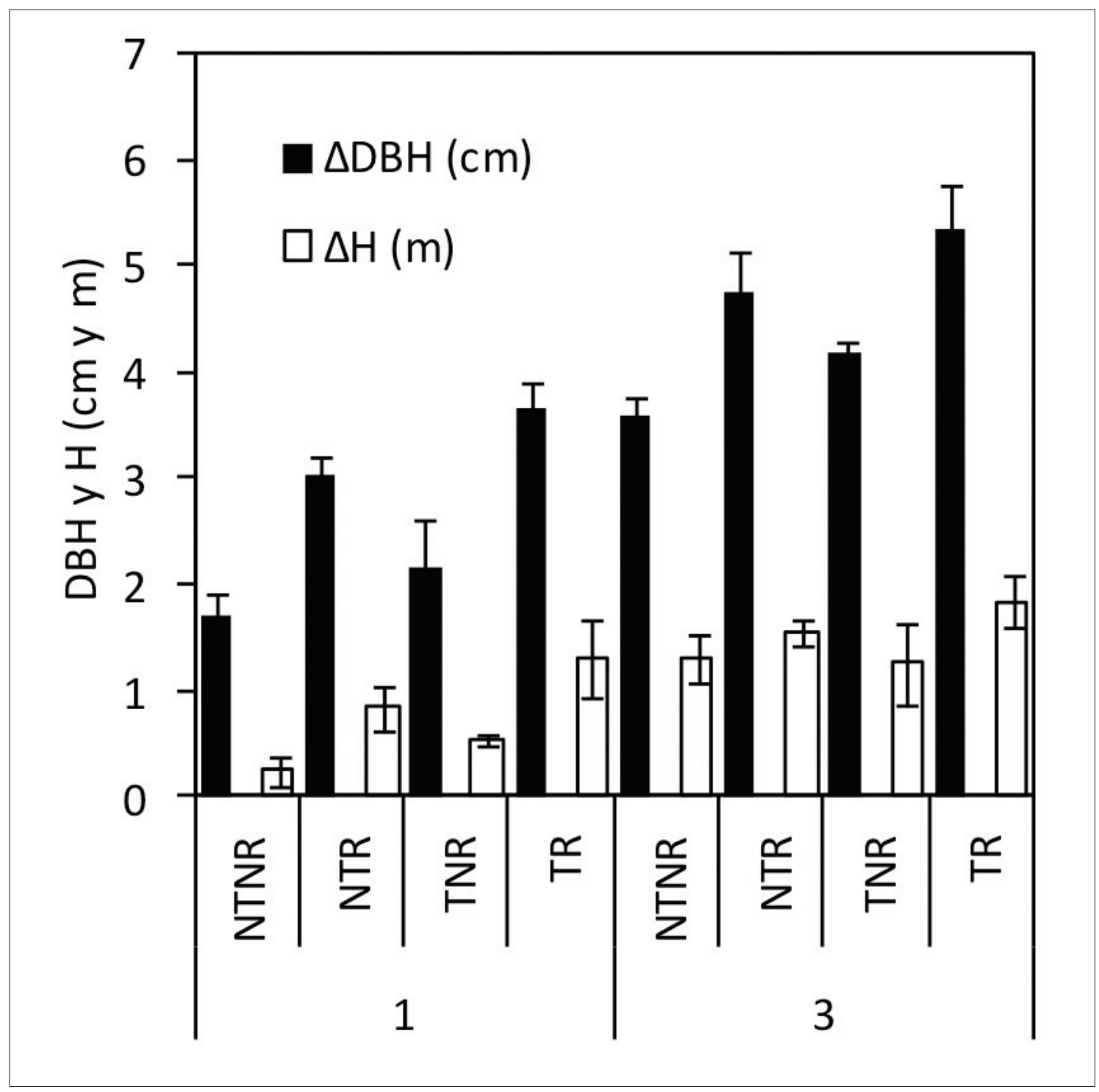

Figura 1. Incrementos de diámetro $(\Delta \mathrm{DBH})$ y altura $(\Delta \mathrm{H})$ para los 3 años de estudio en los diferentes tratamientos (NTNR, NTR, TNR y TR) y según el tipo de suelo (bloques 1 y 3 ). NTNR: suelo no trabajado no regado, NTR: suelo no trabajado regado, TNR: suelo trabajado no regado, TR: suelo trabajado regado.

tamiento no regado ni labrado, en el suelo con poca capacidad de retención de agua (NTNR1) frente al tratamiento regado y labrado en el suelo con una capacidad de retención 3 veces mayor (TR3).

En esta figura se observa el estrés hídrico tan acusado que experimentaron los árboles de cerezo en el tratamiento NTNR1, con pérdidas de hoja ya a mediados de junio para los 3 años de estudio e incluso de mayor magnitud que el efecto producido por la poda durante el primer año. El análisis de la varianza del efecto de los factores estudiados sobre la cantidad sustraída de las copas también indicó un efecto significativo de los 3 factores testados en la materia seca sustraída en los años 2011 y 2013. Las diferencias más notables se encontraron entre los tratamientos NTNR1 y 


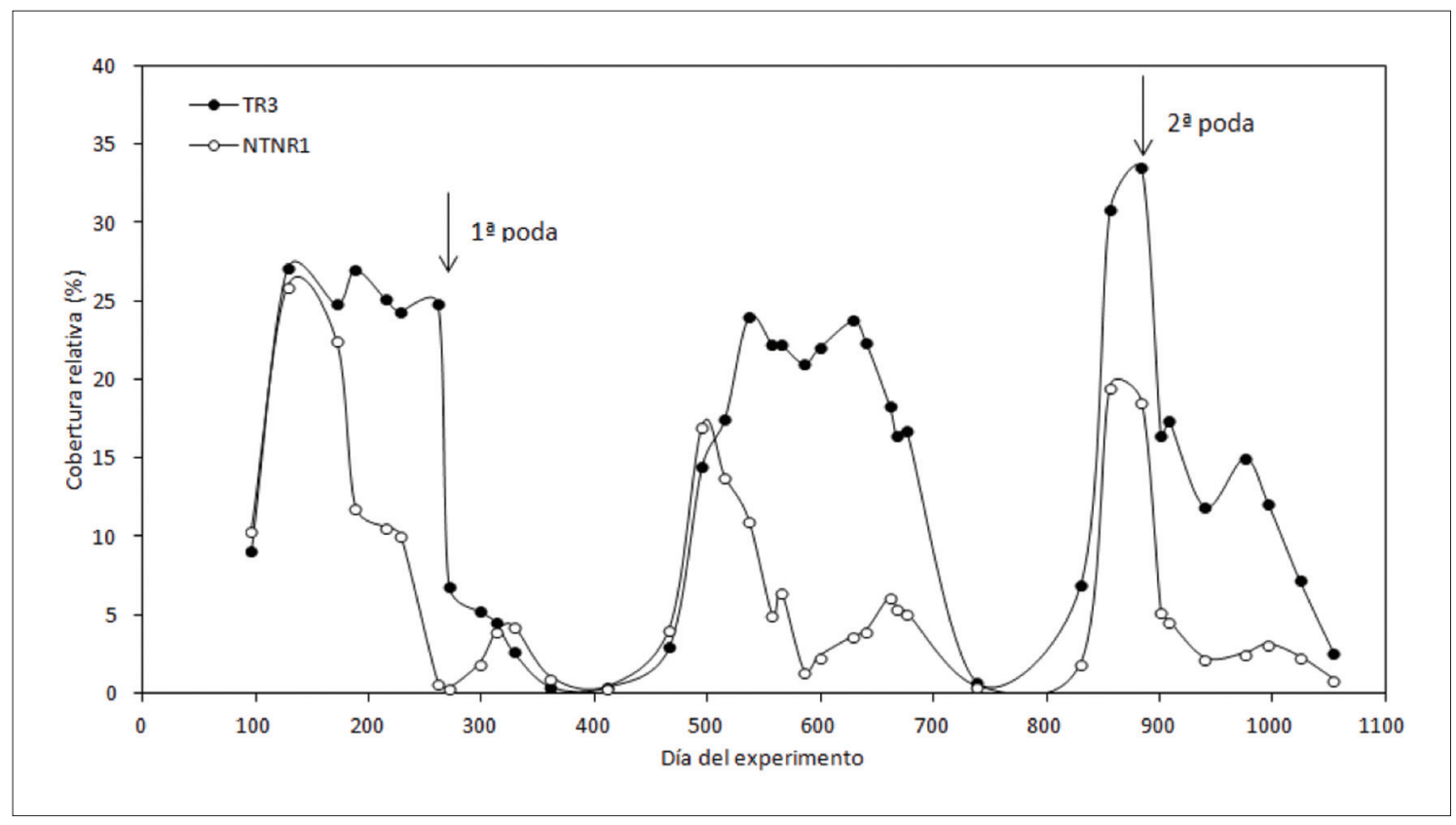

Figura 2. Evolución de la cobertura relativa (\%) durante los 3 años de estudio para los tratamientos NTNR1 (No laboreo+No riego bloque 1) y el TR3 (Laboreo+Riego bloque 3).

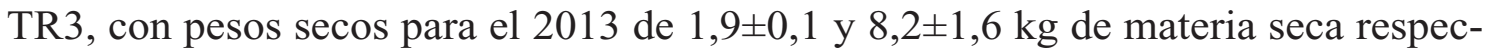
tivamente.

Por último, se observó un efecto significativo del tipo de suelo y del laboreo sobre el contenido de carbono en el suelo para los dos años muestreados, con mayo-

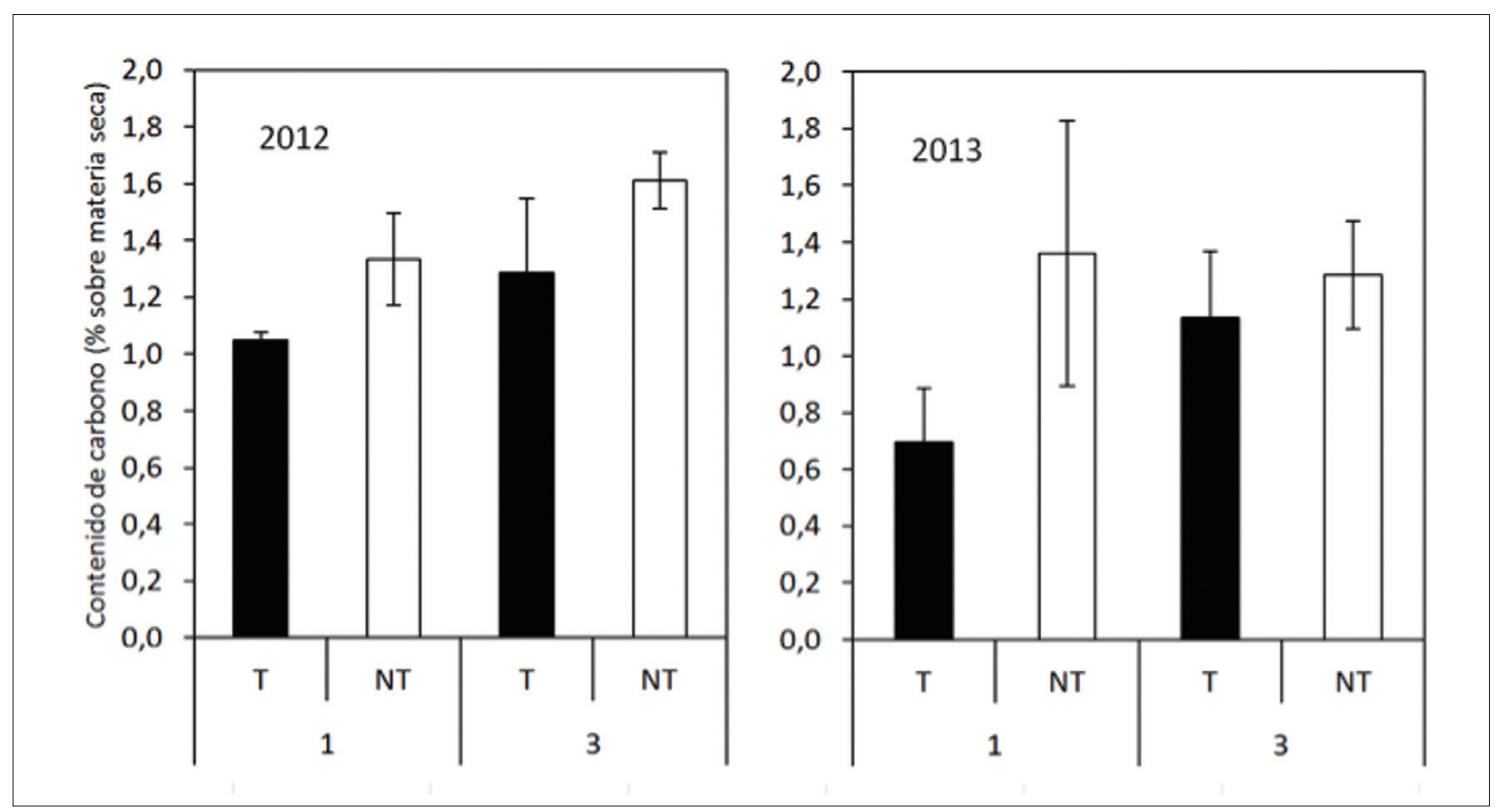

Figura 3. Contenido de carbono (\% sobre materia seca) en el suelo, según tratamiento de laboreo del suelo y el bloque, para los dos muestreos llevados a cabo durante el estudio. T: suelo trabajado, NT: suelo no trabajado. 
res cantidades de carbono a $25 \mathrm{~cm}$ de profundidad en el suelo de mejor calidad agronómica, por un lado, y en las unidades experimentales sin laboreo en el suelo y por tanto con la presencia de vegetación espontánea, por otro lado (Fig. 3). A 50 y 100 $\mathrm{cm}$ de profundidad, por el contrario, no se encontraron diferencias significativas.

\subsection{Ciclo hidrológico}

Durante los 3 años de estudio, la evapotranspiración potencial acumulada ascendió a $2769 \mathrm{~mm}$, mientras que la lluvia fue de $1875 \mathrm{~mm}$. Esta última tuvo un marcado carácter mediterráneo, con escasez de precipitaciones en verano, lo que hizo que las dosis de riego supusieran una media anual acumulada de $296 \mathrm{~mm}$.

En primer lugar, la trascolación supuso un $96 \%$ y la escorrentía cortical un 1.1\% de la precipitación incidente en la parcela rectangular dispuesta para ello. Por tanto, sólo un $3 \%$ de la lluvia quedó interceptada por la estructura forestal y devuelta posteriormente a la atmósfera. Cabe destacar que no se observó diferencia significativa entre los periodos con hojas y los periodos sin presencia de éstas, indicando la escasa importancia de la estructura forestal en la partición de la lluvia.

La Fig. 4 presenta la evolución diaria de la velocidad de flujo de savia para 2 días del mes de Junio de 2013 en las unidades experimentales TR1 y TR3.

Las curvas diarias de velocidad de flujo de savia tuvieron la forma típica de campana, con las velocidades de la parte más interna en general menores a las de la parte externa más próxima al cambium. Sin embargo, en algunos árboles, este perfil decreciente con la profundidad no fue constante durante el periodo de estudio, con incluso mayores velocidades en la parte interna (por ejemplo TR3 en Fig. 4).
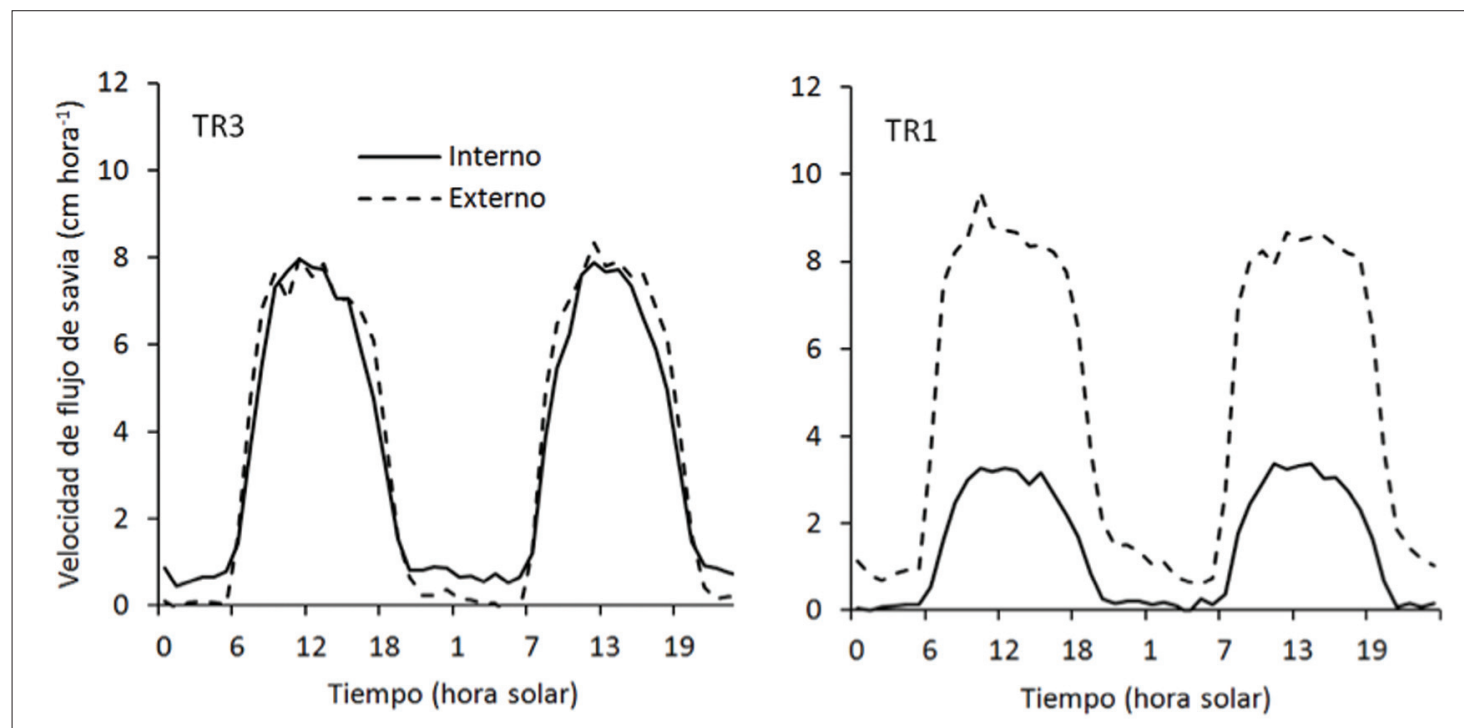

Figura 4. Velocidad de flujo de savia $\left(\mathrm{cm} \mathrm{hora}^{-1}\right)$ para las unidades experimentales TR1 y TR3, en los días 10 y 11 de Junio de 2013. Se presentan las 2 medidas de velocidad realizadas en cada árbol, esto es, la más próxima al cambium (a $1 \mathrm{~cm}$ de éste, externo) y la más alejada (a $3 \mathrm{~cm}$ de éste, interno). 
Tabla 1. Flujo de savia y transpiración acumulados de las unidades experimentales durante los 3 años de estudio. NTNR: no trabajado no regado, NTR: no trabajado regado, TNR: trabajado no regado, TR: trabajado regado.

\begin{tabular}{cccc}
\hline Bloque & Tratamiento & Flujo de savia (1) & Transpiración (mm) \\
\hline & NTNR & 220 & 14 \\
NTR & 896 & 56 \\
TNR & 797 & 50 \\
& TR & 1917 & 120 \\
\hline & NTNR & 2008 & 126 \\
& NTR & 3421 & 214 \\
& TNR & 1939 & 121 \\
& TR & 2877 & 180 \\
\hline
\end{tabular}

Por su parte, los datos acumulados de los 3 años (Tab. 2) desvelaron consumos muy bajos para la mayoría de los árboles testados, correspondiéndose el mayor con $210 \mathrm{~mm}$, equivalente a un consumo diario aproximado durante la época vegetativa de $0.4 \mathrm{~mm} \mathrm{día}^{-1}$ o de 6 litros día $\mathrm{a}^{-1}$. Se observaron efectos estadísticamente significativos tanto del riego como del tipo del suelo $(\mathrm{p}<0.025)$ sobre la transpiración, mientras que el laboreo no la afectó de forma significativa ( $\mathrm{p}=0.25)$.

\section{Discusión y conclusiones}

En este trabajo se han presentado los resultados de un estudio llevado a cabo durante 3 años en una parcela de cerezos jóvenes para madera de calidad ubicada en una región mediterránea, que pretenden, por una parte, servir para evaluar la idoneidad de estas plantaciones en dicho clima, y por otra, valorar cómo los aspectos esenciales del tipo de suelo, riego y laboreo pueden mejorar el rendimiento de la plantación y su comportamiento fisiológico.

Tal y como era de esperar, se han encontrado diferencias significativas en el crecimiento de los árboles entre un suelo más parecido al de ambientes forestales (bloque 1) y un suelo de mayor potencia agronómica (bloque 3).

Los tratamientos no regados tuvieron crecimientos deficientes en el bloque 1, con turnos de corta superiores a 100 años, según las curvas de calidad de estación publicadas en Cisneros (2004). Con la aplicación del riego, el crecimiento en diámetro fue significativamente mayor (media de $1.1 \mathrm{~cm}$ al año), aunque el turno de corta aún estaría alrededor de 70 años. Con respecto al laboreo del suelo, y teniendo en cuenta la unidad experimental con riego por ser la más favorable a la plantación (TR1), su diámetro en 2013 fue de $8,5 \mathrm{~cm}$, de nuevo suponiendo un turno de corta elevado de 70 años.

Los resultados para el suelo franco (bloque 3), sin embargo, representaron en todos los tratamientos turnos de corta inferiores a 55 años, con una reducción apro- 
ximada de éste en 5 años con las prácticas de laboreo y de 10 años con la aplicación del riego. Estos resultados también se pusieron de manifiesto cuando se compararon la materia seca extraída con la poda, así como las dinámicas de la cobertura del dosel, estas últimas indicando estrés hídrico acusado en el suelo franco-arenoso (bloque 1) $\mathrm{y}$ en menor medida en los tratamientos no regados del suelo franco (resultados no mostrados).

Por su parte, el carbono a $25 \mathrm{~cm}$ de profundidad del suelo fue diferente según el laboreo del suelo. Este resultado, sería presumiblemente mayor si se comparasen capas más superficiales donde se concentran la mayor parte de raíces finas de la vegetación espontánea (principalmente leguminosas), tal y como los análisis isotópicos del agua en la planta y en suelo han venido indicando durante el periodo de estudio (resultados no mostrados).

En lo que se refiere a las medidas hidrológicas, la interceptación de la lluvia ha supuesto un valor muy bajo del $3 \%$ de la precipitación, frente a un aproximadamente $10 \%$ esperado para este tipo de plantaciones en ámbitos de similar climatología (por ejemplo, Jackson, 2000). Por un lado, las podas han supuesto una reducción importante en la superficie interceptante. Por otro lado, las características específicas de las hojas de la especie estudiada, esto es, su inclinación pronunciada y su marcada hidrofobicidad, pueden haber tenido también un papel esencial en el escaso efecto jugado por el dosel.

La transpiración de la plantación también ha sido más baja de lo esperado, tanto si se compara con otras plantaciones forestales de densidad y edad similares, como con la misma especie pero para producción de fruto (Juhász et al., 2013).

La reducción de la cobertura como consecuencia de las podas también ha podido jugar un papel fundamental, como recogen otros trabajos donde éstas han supuesto reducciones de hasta un $50 \%$ en el consumo de agua (Clothier, comunicación personal). En lo que se refiere a los efectos testados, el efecto no significativo del laboreo del suelo en el crecimiento y la transpiración de la plantación ha sido el más destacado. En este sentido, el posterior análisis de las dinámicas de humedad del suelo podría desvelar un efecto del laboreo del suelo (tratamientos trabajados) equiparable al aportado por la materia orgánica en superficie y las raíces de las malas hierbas (tratamientos no trabajados), con un supuesto aumento de la macroporosidad del suelo.

En conclusión, los resultados parecen confirmar las indicaciones técnicas para este tipo de plantaciones con manejos intensivos en el ámbito mediterráneo (p. ej., Aletà et al., 2008). Dicho esto, se manifiesta la exigencia del cerezo de condiciones de suelos con capacidades de retención de agua alrededores de $100 \mathrm{~mm}$ y por tanto, de su escasa potencialidad para reforestar suelos forestales de poca capacidad de retención. Con respecto a las principales salidas hidrológicas, la interceptación de la lluvia y la transpiración, han supuesto porcentajes muy bajos de las entradas de agua al sistema (lluvia y riego), de manera que son un indicativo de la gran eficiencia en el uso de agua de esta especie, con aproximadamente 700 litros de consumo de agua por cada incremento en una unidad de diámetro para los tratamientos regados en el suelo franco. Los posteriores análisis a realizar de todas las variables hidrológicas a escalas temporales más detalladas nos brindarán la posibilidad de ser más específicos a 
la hora de dar indicaciones fisiológicas y de manejo para este tipo de plantaciones, incluyendo los coeficientes de cultivo específicos para una correcta planificación del riego.

\section{Agradecimintos}

A.J. Molina es beneficiario de una beca FPI (BES2011-043748) por parte del antiguo ministerio de Ciencia e Innovación, asociada al proyecto AGL2010-21012. Los autores también agradecen la participación en el proyecto Consolider montes (CSC2008-00040).

\section{Bibliografía}

Aletà, N., Vilanova, A., Piqué, M., Coello, J., 2008. Frondoses nobles: Materials vegetals i tècniques de maneig per a la producció de fusta de qualitat. Eds. JM. Tusell y Pau Vericat. Santa Coloma de Farners, Consorci Forestal de Catalunya: 55-63.

Aletà, N., Vilanova, A., 2014. Les plantacions espanyoles de fusta d'alt valor. Catalunya Forestal. 21.

Allen, R.G., Pereira, L.S., Raes, D.,Smith, M.,2006. Crop evapotranspiration-Guidelines for computing crop water requirements-FAO Irrigation and drainage paper 56. FAO, Roma.

Bosch, J.M., Hewlett, J.D., 1982. A review of catchment experiments to determine the effect of vegetation changes on water yield and evapotranspiration. J. Hydrol. 55, 3-23.

Brown, A.E., Zhang, L., McMahon, T.A., Western, A.W. and Vertessy, R.A., 2005. A

review of paired catchment studies for determining changes in water yield resulting from alterations in vegetation. J. Hydrol 310, 28-61.

Burgess, S., Adams, M., Turner, N., Beverly, C., Khan, H., Bleby, T., 2001. An improved heat pulse method to measure low and reverse rates of sap flow in woody plants. Tree Physiol. 21, 589-598.

Cambria, D., Pierangeli, D., 2012. Application of a life cycle assessment to walnut tree (Juglans regia L.) high wood quality production: a case study in southern Italy. J. Clean. Prod. 23, 3746.

Casedesus J., Kaya,J., Bort, J., Nachit, M., Araus. J.L., Amor, S., Ferrazano, G., Maalouf, F., Maccaferri, M., Martos, V., Quabbou, H., Villegas, D. 2007. Using vegetation indices derived from conventional digital cameras as selection criteria for wheat breeding in waterlimited environments. Ann. Appl. Biol. 150, 227-236.

Cisneros, O., 2004. Autoecología del cerezo de monte (Prunus Avium L.) en Castilla y León. Tesis Doctoral. Universidad Politécnica de Madrid.

Farley, K.A., Jobbagy, E.G., Jackson, R.B., 2005. Effects of afforestation on water yield: a global synthesis with implications for policy. Glob. Change Biol. 11, 1565-1576.

Gliessman, S.R., 1990. Agroecology: researching the ecological basis for sustainable agriculture. In Gliessman, S.R (Eds), Ecological Studies. 78. Springer Verlag. pp. $3-10$. New York. 
Jackson, N.A., 2000. Measured and modelled rainfall interception loss from an agroforestry system in Kenya. Agr.For.Meteo.100, 323-336.

Josa, R., Quintana, A., 2000. Torre Marimon: Una pequeña cuenca en el medio agrario mediterráneo. Cuadernos de Investigación Geográfica.26, 67-80.

Juhász, A., Sepsi, P., Nagy, Z., Tökei, L., Hrotkó, K., 2013. Water consumption of sweet cherry trees estimated by sap flow measurement. Scientia Horticulturae. 164, 41-49.

MAPA (eds.), 2006. Forestación de Tierras Agrícolas. Análisis de su evolución y contribución a la fijación de carbono y al uso racional de la tierra.

Montero, G., Cisneros, O., Cañellas, I., 2003. Manual de selvicultura para plantaciones de especies productoras de madera de calidad. INIA, Madrid.

Moreno, F., Pelegrín, F., Fernández, J.E., Murillo, J.M., 1997. Soil physical properties, water depletion and crop development under traditional and conservation tillage in southern Spain. Soil Till. Res. 41, 25-42.

Ripoll, M.A., Sánchez, A., Gallego, E., Jiménez, M.N., Navarro, F.B., 2013. Proyecto INIA 2008-00004-00-00 “Optimización del riego en cultivos de frondosas destinadas a la producción de maderas de calidad mediante la utilización de indicadores del estado hídrico del arbolado y del suelo": actividades realizadas y resultados obtenidos.

Umebayashi, T., Utmusi, Y., Koga, S., Inoue, S., Shiiba, Y., Arakawa, K., Matsumura. J., Oda, K., 2007. Optimal conditions for visualizing water-conducting pathways in a living tree by the dye injection method. Tree Physiol. 27, 93-99. 